\title{
Impaired sensorimotor integration in Parkinsonism and dyskinesia: a role for corollary discharges?
}

\author{
A P MOORE \\ From the Glasgow University Department of Neurology, Institute of Neurological Sciences, Southern General \\ Hospital, Glasgow, UK
}

SUMMARY In a bimanual slow matching task patients with asymmetric bradykinesia overestimated the movement of the more bradykinetic limb. Patients with drug induced or idiopathic asymmetric dyskinesia or dystonia, and patients with unilateral arm weakness underestimated movement of the abnormal limb. Bradykinesia may be caused by reduction, and dyskinesia and dystonia by exaggeration of corollary discharges.

The movement disorder in Parkinson's disease encompasses a variety of phenomena including abnormalities of initiation, maintenance, accuracy, velocity and extent of active movement, and resistance to passive movement. We lack a unifying explanation of these disparate factors and experiments exploring one feature often ignore others, although concepts derived from such investigation may advance a general theory; in this paper the reduced extent of active movement (strictly, hypokinesia) is considered, but the more familiar term "bradykinesia" is used for convenience.

The role of the basal ganglia in the control of movement is unclear, as is the mechanism by which basal ganglia hypofunction results in bradykinesia. Marsden has argued that the basal ganglia are probably not primarily involved in initiating movement or the selection of specific muscles, and suggests that they play a role in scaling the amplitude of movement. ${ }^{1} \mathrm{He}$ proposed that in bradykinesia there is a failure of "automatic execution of learned motor plans", and that although there are abnormalities of proprioceptive feedback, kinaesthetic function is not disturbed and sensory input does not influence the generation of bradykinesia; rather, there is a simple failure to generate an adequate motor signal. ${ }^{1}$ Hassler, however, points out that there is extensive indirect sensory input to the striatum ${ }^{2}$ and indeed sensory and other cognitive abnormalities are described in Parkinson's disease. ${ }^{3}$ Their mere presence is insufficient evidence that sensory abnormalities promote

Address for reprint requests: Dr A P Moore, Department of Neurology, Southern General Hospital, Glasgow G51 4TF, UK.

Received 29 November 1985 and in revised form 30 July 1986. Accepted 23 September 1986 bradykinesia and in many patients there is a striking discrepancy between motor deficits and intact sensory and cognitive function. ${ }^{1}$ Nevertheless, the possibility remains that they are important.

If there is simply a failure to generate an adequate signal, then how does bradykinesia differ from weak ness? A patient with weakness is able to detect the reduced power and, within limits, can compensate byo voluntarily increasing effort. One explanation of this is that when the brain issues a motor command it keeps a record, a "corollary discharge", of the command. Subsequently by comparing this record with the force actually generated (as signalled by peripheral feedback) the brain automatically detects any discrepancy and attempts to correct it. The principle is illustrated in figure 1. McCloskey et al ${ }^{4}$ and Matthews ${ }^{5}$ discuss the evidence that the brain can use the corollary discharge itself as a measure of effort expended, without reference to peripheral feedback. However, in judging the success of active movement as opposed to effort, it is likely that the brain requires both corollary discharges and peripheral feedback. ${ }^{45}$

Rapid movements made without visual guidance have been called "open-loop" with proprioceptive feedback not available in time to modify the movement. Slow movements without vision are probably "closed loop", the corollary discharge being compared with proprioceptive signals. Goodwin et al established that the brain is able to and usually does use the combination of corollary discharges and sensory feedback to gauge slow movement, but it remains possible that feedback alone may suffice. It is unlikely that the strategy of employing both feedback and corollary discharge would be abandoned as it is probably essential in distinguishing passive frcm active movement and this distinction needs to be 
Normal

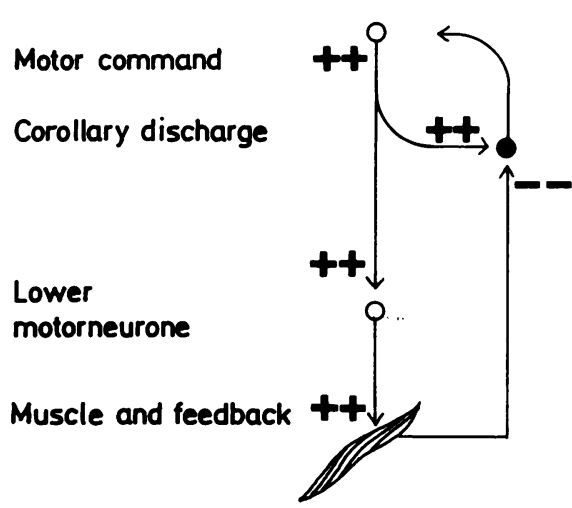

(UMN) Weakness

Bradykinesia

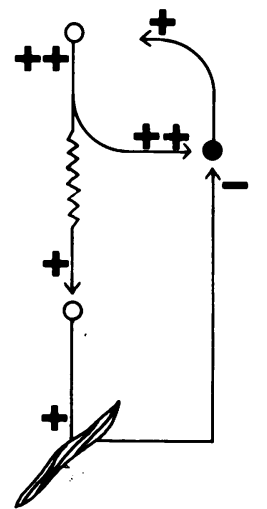

Dyskinesia / dystonia
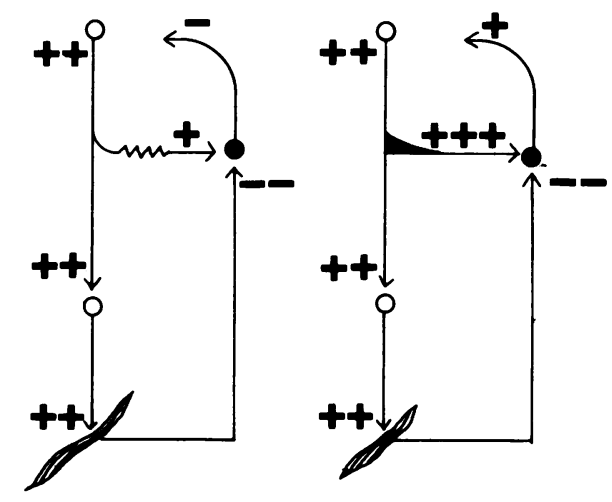

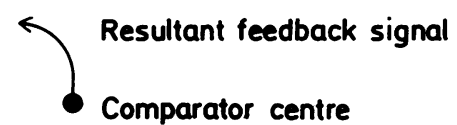

Fig 1 Diagram to illustrate the principle of corollary discharges. Each stage is not necessarily monosynaptic. Normally the motor command passes unaltered through the system to muscle, simultaneously giving off a corollary discharge (CD) of equivalent strength. This passes to a comparator which correlates it with returning afferent signals, and the resultant, a kind of error signal, passes back to the motor command and is used to adjust the movement. It may also influence perception. In weakness the mismatch betweeen the full strength $C D$ and afferent signals gives a positive resultant. This boosts the motor command which in turn generates an increased CD. At the conscious level the increased CD conveys a sense of increased effort. In bradykinesia the CD may be diminished. The comparator perceives more effect than was required and its resultant tends to reduce the motor command. In dyskinesia the CD may be exaggerated; the comparator finds less effect than intended, and the resultant boosts the motor command. See text for further discussion.

made continuously.

In weakness it seems that the relevant comparison of corollary discharge and peripheral feedback occurs at a conscious level (here arbitrarily labelled the "cortical" level). It is possible that similar comparisons occur at a subconscious level. Failure of such a mechanism could result in bradykinesia if errors are undetected and therefore cannot be corrected.

Hughlings-Jackson first proposed multilevel "hierarchical" systems of motor control ${ }^{7}$ and this remains an important though much modified concept. $^{8}$ In such schemes, higher tiers of control undertake more general, more conscious and volitional decisions, directing the activity of lower tiers which process more specific details. Each level receives information about evolving motor commands, the state of other levels, and ascending sensory signals. In concert with this idea, Marsden's proposal that the basal ganglia "automatically execute learned motor plans" 1 implies that a separate executor is available for other situations, notably new or difficult tasks where perhaps the "cortical" system is employed.

There have been many tracking experiments in bradykinetic patients. ${ }^{19-11}$ Most involve subjects tracking an external signal with or without visual feedback, often with speed of response an important factor.
Many are novel situations allowing, even requiring, constant "cortical" intervention with the matching of information from separate sources, such as vision and proprioception. This paper describes a matching experiment to investigate perception using a task which attempts to involve only the more "automatic" systems of the basal ganglia. Such a task should be simple and preferably exclude vision, making an external reference signal inappropriate. As far as possible a single sensory modality should be used. Movement speed should be such that slowed motor output affects matching ability as little as possible.

These conditions can be approached if a subject with unilateral or sufficiently asymmetric bradykinesia attempts to match the active movement of one arm with the other, without vision or severe time constraints. He compares proprioceptive feedback from each limb and the cross-modality process of matching proprioception with vision is avoided. The normal limb acts as an intra-individual control.

In this paradigm the subject continually compares his assessments of the success of motor commands to each limb, and if one assessment is defective then a discrepancy will arise between the movements of the two limbs. A discrepancy will arise even if the defect is only relative, both arms being abnormal to different 
degrees. If the limbs are equally abnormal then no discrepancy would be expected. Effectively, each limb is tracking the other.

\section{Subjects and methods}

Twenty two patients, mean age 59 years, with mild or moderate idiopathic Parkinson's disease (Hoehn and Yahr grades I to III) were studied. They all had predominant bradykinesia and the majority had unilateral or markedly asymmetric disease. Their drug schedules were not altered. Three were studied during a period of dyskinesia of the usually bradykinetic arm, the other limb remaining normal.

Four patients with mild unilateral arm weakness (MRC grade IV), either upper or lower motor neuron, who had no sensory symptoms or sensory loss detectable by conventional clinical examination were also studied, as were three patients with unilateral idiopathic dystonia.

Twelve siblings or spouses of these patients, mean age 52 years, were recruited as control subjects. All subjects gave informed consent.

The severity of Parkinson's disease was estimated using the Webster (0-30) and Hoehn and Yahr (0-5) rating scales.

The symmetry of bradykinesia was graded on a five point scale from strongly left to strongly right sided disease. A more objective measurement of symmetry called the bradykinesia laterality score (BLS) was obtained using a battery of tests completed separately with each arm.

Each test was performed as fast as possible for 20 seconds, and received roughly equal weighting by counting the sum of

(1) half the number of taps of the thumb tapping each ipsilateral finger in turn

(2) the number of cycles of alternate tapping of the dorsum and palm of the hand on the ipsilateral thigh

(3) the number of taps of the index finger going between two marks $50 \mathrm{~cm}$ apart on a table.

$$
\text { BLS }=\left(\frac{\text { score for the "good" hand }}{\text { score for the more affected hand }}-1\right) \times 100
$$

It tested both distal and proximal movements, and accurately reflected clinical impressions of bradykinesia symmetry. However, scores for individual arms did not correlate well with clinically estimated bradykinesia.

\section{Procedure}

The subject was seated comfortably with elbows in front of him resting on a padded table. Each elbow was aligned with the axis of rotation of a metal rod which was strapped to the wrist. The arm was in the most comfortable position of mid pronation-supination. Potentiometers at the axes of rotation allowed the angles of flexion-extension of each arm to be continuously recorded. The apparatus had sufficient damping to allow good end points to be read despite moderate tremor or dyskinesia, though the influence of these could easily be discerned.

With eyes open subjects were familiarised with the apparatus and with each task individually. The subject was asked to flex and extend both elbows together, maintaining the same velocity and range of movement and keeping the forearms aligned. Flexion always followed extension and viceversa, with time allowed between movements to correct any discrepancies noticed by the subject.
In self-matching modes, subjects followed verbal commands to start and stop or to move faster or slower, and moved both arms freely. In wrist guided modes one or other wrist (the reference arm) was lightly grasped by the experimenter and guided as smoothly as possible. A gentle pull was applied to signal the start and end of each movement. Care was taken only to guide the angular velocity of the reference arm, by light touch on the back or front of the wrist, with subjects providing the motive power to ensure active, not passive, movements. Subjects followed with the other (tracking) arm. They were verbally warned of the approximate velocity and of impending starts and stops which were executed gently to avoid surprise.

Both self-tracking and wrist guided trials with each arm were performed twice, once at a faster $\left(20-40^{\circ} . \mathrm{s}^{-1}\right)$ and once at a slower $\left(2-5^{\circ} . \mathrm{s}^{-1}\right)$ speed. Even the fast speed was well within the capabilities of the slowest of the patients, and this was checked by performing a few faster movements at the end of each fast trial.

A predetermined sequence of trials was followed, each trial being preceded by 5-10 practice movements with eyes open, practice continuing until the experimenter was satisfied that the subject understood the task. Measurements were then made with eyes closed. The sequence was Sf, Ss, Lf, Rf, Ls, Rs, where $S=$ self-matching, $L / R=$ left $/$ right arm tracking, $\mathrm{f} / \mathrm{s}=$ fast $/$ slow movements. Each trial consisted of 10 movements using one combination of tracking mode and speed range. Individual movements were main tained at an approximately constant velocity, but the veloc ity and excursion were altered between movements in random manner (within the speed range). Only measurements in which both arms moved at least $30^{\circ}$ were con sidered valid because the use of displacement ratios in the analysis might produce undue bias if movements, although producing a small disparity in terms of position, happened to have a large gain (see analysis).

\section{Comment on technique}

At the end of each movement the subject had to decide where to stop the tracking arm. If any lag remained uncompensated because of difficulty generating movement as opposed to detecting it, then the choice of cue by the subject would be crucial in determining the end point of the lagging side. In order to avoid confusion, initial instructions to subjects were simple, and therefore ambiguous. They could have used either velocity, timing, displacement or position matching to move both arms together. As the practice session proceeded, subjects were encouraged primarily to match displacement. In practice most subjects did this spontaneously but still some might have used the timing of the halt of the reference arm as their cue to halt the tracking arm. Using end point measurement, a slower bradykinetic arm would not then indicate the perceived range of movement of a faster, normal, reference arm. This was the main reason for also recording when the good side was tracking the bradykinetic (reference) arm. Such errors would be reversed and the mean of the two gains should reflect perception. In this context "perception" is applied to the resultant feedback (fig 1) as opposed to the raw proprioception unmodified by the corollary discharge.

Analysis Recordings were inspected visually and measurements were made of the end points of each movement, 
allowing the displacement of each arm to be calculated. For each movement the ratio was calculated of the larger displacement to the smaller and expressed as a percentage increase called the "gain". This represented the percentage error of matching.

For controls the gain was deemed positive if the right arm moved further, negative if the left moved further. The sign of patients' gains was arranged so that greater displacement of the good arm gave a positive gain. Mean gains were calculated for each subject, type of disorder, mode of trial and various aggregates such as all normal subjects or all bradykinetic patients, bradykinetic/normal limb tracking or selfguided trials, fast/slow, etc and were compared using Student's $t$ test. Data from all the trials with each individual were pooled to calculate a mean overall gain for that individual.

\section{Results}

\section{CONTROLS}

Inspection of traces (fig 2) Even the more accurate control subjects frequently misaligned their arms by 5-10 (maximum $18^{\circ}$ ) at the end of the movement. Despite this, displacement of one arm tended to be maintained within a few degrees of the other for many cycles, producing a relatively consistent end point error, although the end point error would wax and wane between $+10^{\circ}$ and $-10^{\circ}$.
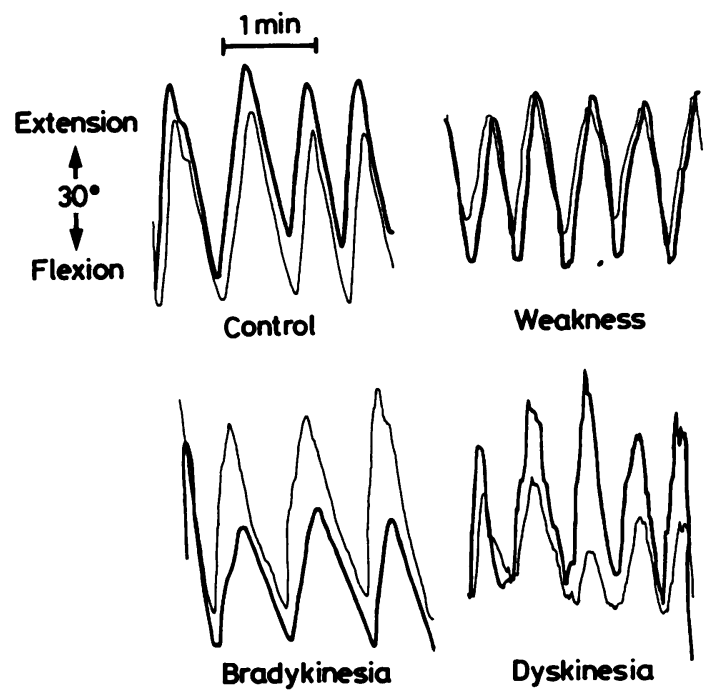

Fig 2 Traces obtained from normal controls and patients with unilateral weakness, bradykinesia and Parkinsonian drug induced dyskinesia. The traces are slightly out of step in time. For control traces, the thick line is from the right arm. For patients the thick line is from the more abnormal arm. These are "slow" movements. Traces showing consecutive series of errors are shown for clarity but were unusual. In some of the movements illustrated one limb moves less than $30^{\circ}$ : these movements were not included in the statistical analysis.
For most control subjects, vision made little difference to the ability to match displacement. Indeed, some performed more accurately with eyes closed, perhaps because of better concentration. Without vision end-point errors were greater.

During slow movements subjects reported their attention shifting from one arm to the other, with continual fine adjustments. In wrist guided movements there was often a momentary lag in initiation of tracking, but subjects equally often seemed to anticipate and move in advance of the reference arm. After a brief mismatch the error was generally compensated.

Statistical comparisons (fig 3) For both the eight right-handed and the four left-handed control subjects there was an apparent tendency for the right arm to move further, with a combined mean gain of 1.86 . This was thought to represent a centring error of the apparatus and an allowance was made for this in comparisons with patients. There was no significant difference between right and left handed control subjects overall or in individual modes.

Control subjects matched displacement very accurately in self-matching trials (mean gain 1.9). In wrist guided tracking they showed a small but significant tendency for the tracking arm to move further, as shown by the difference between gains for tracking with the right arm (gain 4.03) and the left arm (gain -0.03 ), which was statistically significant, $p<0.01$. This was mainly evident during fast tracking. In slow tracking the use of right or left as the tracking arm made no significant difference.

\section{PATIENTS}

There was a roughly normal distribution of gains. There was some skewness not considered sufficient to prevent the use of parametric statistics, although conclusions did not alter when non-parametric statistics were used. There was no difference between right and left handed controls so no allowance was made for handedness in patients.

Inspection of traces (fig 2) There were obvious differences from control subjects although patients were often just as accurate, especially with eyes open. Often a trial would commence with accurate matching which was sometimes maintained throughout. When this happened patients remained prone to the same mistakes as control subjects, with an end-point error being maintained for several cycles, but commonly they began to make systematic errors of displacement matching. Errors might appear immediately or after a few movements and be intermittent or sustained over several movements. They were no more common towards the end of a trial and there was no fatigue or learning effect. They occurred in all types of trial. 
Normal controls

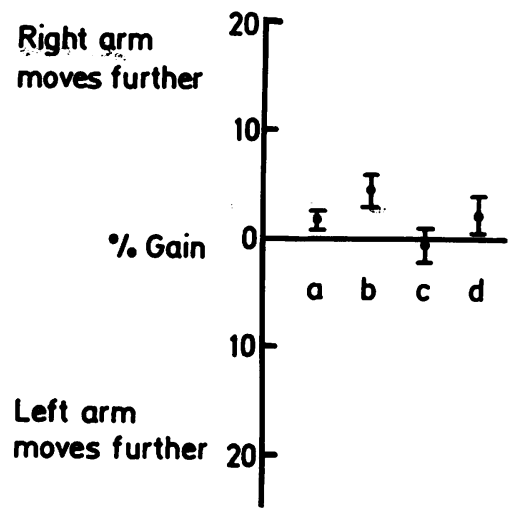

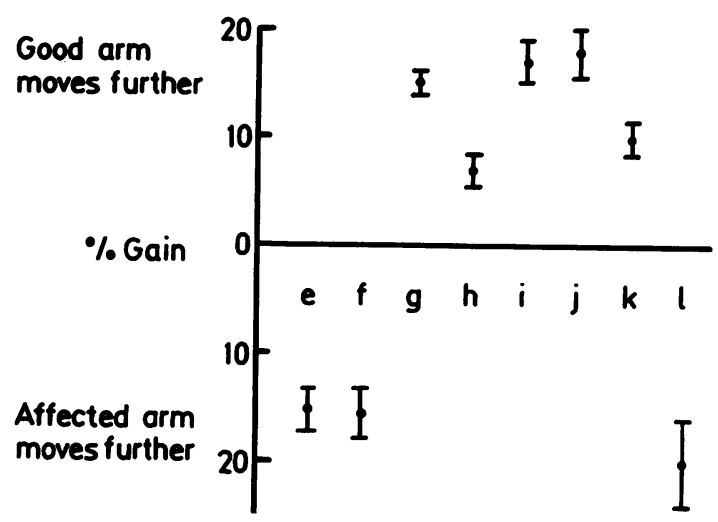

Parkinsonian patients

Subjects

$\begin{array}{ll}\text { Subjects } & \text { Modes } \\ \text { a normal. } & \text { all } \\ \text { b normal } & \text { right tracking } \\ \text { c normal } & \text { left tracking } \\ \text { d normal } & \text { self tracking } \\ \text { e weakness } & \text { all } \dagger \\ \text { f dystonia } & \text { all } \dagger\end{array}$

g All bradykinetic

h Untreated bradykinetic

i All bradykinetic

j All bradykinetic

$k$ All bradykinetic

I All dyskinetic

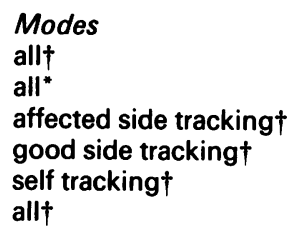

Differences from normal subjects: ${ }^{*} p<0.01 \dagger p<0.001$

When one side lagged most patients noticed when the disparity was greater than $10-15^{\circ}$ (usually less) and partly corrected, only to begin falling behind again. This threshold was inconstant. With vision, patients did not allow large disparities to develop. Commonly, in the trials in which the good arm was tracking the bradykinetic reference arm, traces showed that the overshoot of the good arm was partly corrected after the reference arm halted. Thus the timing of the halt-of the reference arm was not the major cue for halting the tracking limb, and it is unlikely that patients were using corollary discharges alone to match limb movement.

The following patterns emerged:

(a) In symmetrical bradykinesia, displacement matching remained accurate with errors just as likely to occur in either direction, though generally larger than controls. In asymmetric bradykinesia the affected side tended to lag in all modes, though this was inconstant and on occasion it moved further.

(b) In Parkinsonian patients tested in both phases, the affected arm moved through a smaller range in bradykinesia but through a larger range when it was dyskinetic, the other arm remaining normal. Only two subjects underwent this transition, and not all modes were examined in each state partly because the transition was clear from inspection of the traces, and partly because the session could not be extended. A third Parkinsonian patient with persistent drug induced unilateral dystonia and two subjects with both idiopathic torticollis and dystonia of one arm also moved the affected arm through a larger range. Another with torticollis but only a history of arm dystonia did not.

(c) In upper or lower motor neuron weakness, patients moved the weaker arm through a greater range.

Statistical comparisons (figs 3,4)

(a) Patients with asymmetric bradykinesia.

(1) Comparison of mean gains for patients with right sided disease (mean gain 15) and left sided disease (mean gain 16) showed no significant difference between them: both moved the affected side through a smaller range, and this occurred in all modes.

(2) If the affected side was tracking the good (reference) arm, the affected side lagged, on average by $17 \%$.

(3) More remarkably, when the good side was tracking the affected (reference) arm, the good arm still moved further, on average $18 \%$.

(4) During self-matching trials the good side moved further, by $10 \%$. Each of these findings differed from controls, $p<0.001$. The mean overall gain for each bradykinetic patient was related to the bradykinesia laterality score $(r=0.84, p<0.001)$ (fig 4$)$. 


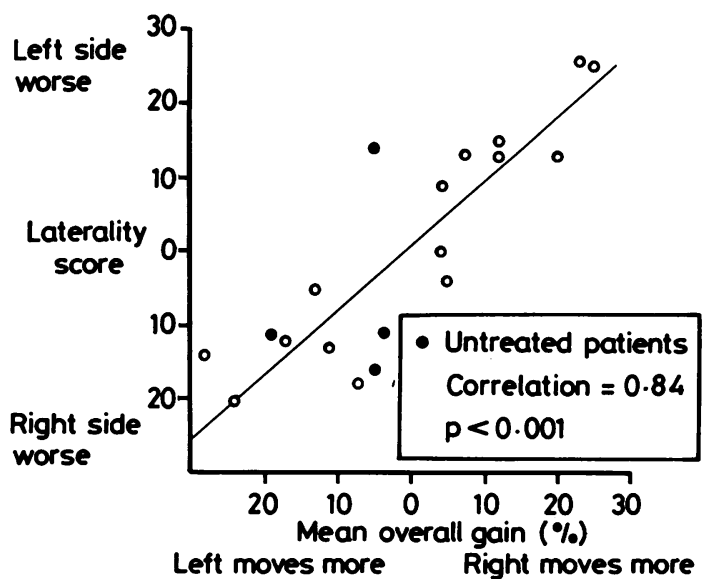

Fig 4 Correlation between symmetry of bradykinesia (BLS) and mean gain (all modes) for individuals with bradykinetic Parkinsonism.

(5) Patients on antiparkinsonian agents displayed much larger gains (mean 15) than four untreated patients (mean 6), p < 0.01, despite comparable mean bradykinesia laterality scores of 14.4 and 13.8 respectively. Both differed from controls. Three further untreated Parkinsonian patients with asymmetric bradykinesia were tested, and the mean gain for the total of seven patients remained at $8 \cdot 2$.

(6) As assessed clinically, the severity of bradykinesia in patients with more asymmetric disease did not differ significantly from the severity in bilateral disease.

(b) Patients with dystonia, dyskinesia or weakness

(1) The combined mean gain for three Parkinsonian subjects with unilateral dyskinesia was -20 , and for three patients with idiopathic dystonia was -17 , both significantly different from normal $(p<0.001)$. Thus the abnormal limb tended to move further than the normal limb.

(2) The mean gain for patients with arm weakness was -14 , so that the weak arm regularly moved further.

\section{Discussion}

The results of this experiment suggest that in bradykinesia there is difficulty either in detecting what proportion of a required movement has occurred, or in using this information to modify the motor programmes. Although the results are compatible with "failure to automatically execute learned motor plans", "they also point to defective sensory and motor integration as an important element in that failure.

Corollary discharges can provide the missing link. Three types of error could account for these findings: (a) corollary discharges are reduced (b) afferent signals are increased

(c) the integrating unit is defective.

\section{(a) Reduced corollary discharge (fig 1)}

If the corollary discharge is reduced or does not receive its full weighting in the comparator unit, then the brain would interpret the movement of the bradykinetic arm as excessive and consequently would reduce the motor command. This need not disrupt the form of the command, only its extent. If such an error can be provoked by dopamine deficiency, then dopamine excess might cause the reverse; corollary discharges might be exaggerated. The system would then automatically increase motor commands.

\section{(b) Increased afferent input}

The same result may be produced by modification of the returning sensory input rather than the corollary discharge. Tatton et al proposed an equivalent mechanism to account for both rigidity and bradykinesia ${ }^{12}$ but their data showing exaggerated long-loop reflexes in Parkinsonian rigidity could equally be due to diminished corollary discharges. No experiment has yet distinguished between the effects of exaggerated sensory input and reduced corollary discharge.

Attempts were therefore made to employ purely passive movements in the reference arm, but subjects found it difficult to avoid helping the movement and hence introducing an unknown corollary discharge. Experiments on tracking illusions of movement induced by muscle vibration are proceeding to clarify this point. Such vibration sets up muscle spindle derived signals which are interpreted involuntarily by the brain as passive movement of the joint served by that muscle. This is thought to be because no corollary discharge is available to match the sensory signals (as it would be in active movement). ${ }^{6}$ The occurrence of larger vibration induced illusions in a bradykinetic limb would point to abnormalities of the afferent arc.

A delay in sensory feedback has been reported in Parkinsonism, ${ }^{13}$ though never confirmed. The measured delay was between a visual and a tactile stimulus, involving cross-modality integration, and was of the order of $70 \mathrm{~ms}$, probably too small to influence the slow matching task described here.

\section{(c) Defective integrating unit}

Corollary discharges or returning sensory input could either be distorted before they reach the comparator centre or be inappropriately weighted on arrival there. There is evidence that both mechanisms can operate. ${ }^{8}$ A subtractive operation using unweighted signals is the simplest use of corollary discharges and sensory input. An increase in corollary discharges would be directly equivalent to a decrease in sensory 
input. Mathematically, however, other ways of integrating the two signals are feasible, as well as weighting one or other, so that they are not necessarily equivalent.

\section{Hierarchies in motor control}

In weakness the corollary discharge may still pass normally to a comparator centre where it is integrated with afferent signals showing less effect than required. At first sight the resultant signal appears to be the same in both dyskinesia and weakness, and for conscious movement there remains the strategy of increasing the motor command. However, there may be separate tiers of control operating at different stages in the evolution of a motor command. Two or more feedback systems may interact, possibly employing anatomically and biochemically distinct comparators and with different levels of access to consciousness.

Thus an error signal arising from a higher "cortical" system might reach consciousness. If the subject becomes aware of weakness he is able to increase the effort voluntarily and in a controlled manner. If a similar error signal arose at a lower tier of control which did not reach consciousness then fluctuations would not be consciously perceived or subject to voluntary compensation, reflecting the situation in dyskinesia and bradykinesia.

Cools $\mathrm{et}^{\mathrm{B}} \mathrm{l}^{8}$ have proposed a similar mechanism in which a hierarchy of feedback systems involves the basal ganglia in sensorimotor integration. A decision to move (or raw motor command) at the top is progressively modified along a series of comparator centres, each centre taking particular account of different kinds of information. Each centre receives the descending motor command, called the "reference" signal, from superior hierarchies, and an ascending afferent input such as proprioceptive data, and produces an output. The output acts as the reference signal for lower tiers and so on until all the relevant influences are accommodated and the command passes to the muscles. The motor command or reference signal doubles as a corollary discharge, and the output also functions as the "resultant" in fig 1.

Both descending motor commands and ascending sensory input may be able to by-pass a given level, allowing the system to produce some motor output even if one level is disrupted, although characteristic disorders of movement may occur. A distortion introduced at one level may be detected if the output from that level is not compatible with output from higher tiers which has by-passed the abnormal level. If the distortion remains undetected it will influence the motor act. If it is detected then it may be possible to over-ride it or to promote the more appropriate signal. This implies surveillance of the success of each hierarchical tier.

In broad terms, the "automatic" basal ganglia level is more likely to be involved in easy, familiar, repetitive acts requiring little concentration. A certain degree of difficulty such as in learning a new task may cause engagement of greater "cortical" control which is slower and requires more conscious attention. Cross-modality sensory integration may make "cortical" involvement more likely, and this might account for the visual facilitation (classically of gait) seen in Parkinsonism.

Thus two findings of the present experiment might be explained: the intermittent nature of the gain error and the difference between gains measured in asymmetric bradykinesia in treated and in untreated patients. As tracking proceeds, "automatic" basal ganglia control may normally be used in preference to "cortical" systems, whilst remaining under surveillance. During a phase of basal ganglia predominance, dysfunction may cause an error of gain. If the error is detected the "cortical" system (which is still accurate) takes over and compensates, so that gain reverts to zero.

The ability to compensate for error depends on successful surveillance of movement accuracy. In treated patients two factors militate against this. First, a given degree of bradykinesia occurring in a treated patient implies more severe underlying disease thang when it occurs in an untreated patient. Second, whils it may well be improving basal ganglia function, "smokescreen" of exogenous dopamine is present in" treated patients and may be acting at abnormal sites or preventing physiological modulation of dopaminergic tonus, although this is speculative. Both factors could impair the surveillance of movement accuracy and change the threshold of difficulty or error required to engage cortical control. Treated patients would more persistently rely on faulty basal ganglia control resulting in a higher mean gain than in untreated patients. Experimental procedures which allow less "cortical" intervention might prevent this.

In this context the finding that a weak limb tended to move further than its normal fellow was unexpected and difficult to explain. It is possible that the lesions causing weakness (two strokes, two brachial plexus lesions) also caused sensory abnormalities not detected clinically.

\section{Peripheral or central feedback loops?}

Ballistic movements are generally regarded as "openloop" because there is insufficient time for peripheral feedback to influence the movement. The diminished ballistic movements seen in bradykinesia have therefore been explained in terms of reduced motor commands alone. ${ }^{1}$ It is more difficult to invoke abnormalities of sensorimotor integration as the 
cause of this aspect of bradykinesia.

However, if sensorimotor integration could use feedback systems within the brain then the time required for some loops would be substantially reduced. A descending motor programme may be compared with pre-existent ascending afferent signals. ${ }^{8}$ Consequent modifications to the motor signal may be commenced before it leaves the brain. In support of this, tracking experiments have suggested that errors of direction of movement can be corrected in a time too short for returning afferent signals to have any influence, probably by comparing the final motor output to the periphery with the initial command: an internal feedback loop. ${ }^{10}$ Secondly, in estimating the time of onset of an active movement, normal subjects appear to use central command-related signals in preference to sensory feedback from the moving part. ${ }^{4}$ Thirdly, motor commands insufficient to activate even the earliest recruited fibres of a muscle, and hence (probably) not provoking afferent feedback, can be shown to influence the threshold at which electrical stimulation of motor cortex activates these fibres. Such subthreshold commands can be maintained for at least 5-10 seconds, and hence may be monitored and adjusted without recourse to peripheral feedback. ${ }^{14}$

\section{Evidence from single neuron recordings}

In the pallidum, the output tract of the basal ganglia, single neuron recordings have shown that cell firing correlates well with active movement (equivalent to the motor command), and less well with sensory input, ${ }^{1}$ suggesting that the basal ganglia might lie upstream of the comparator centre. However, a significant proportion of the neurons in the pallidum and particularly in the putamen (the basal ganglia input) which are related to active movements do respond to passive somatosensory stimulation of the same body part, ${ }^{15}$ so that a comparator centre could be in the basal ganglia.

Dopaminergic cells may determine the weighting of corollary discharges in either case. Dopamine probably acts at the synaptic level by reducing the effect of glutamic acid as a neurotransmitter. ${ }^{16}$ Corticostriate cells use glutamate and fire in a manner phasically related to active movements, consistent with the carriage of a motor command or corollary discharge. ${ }^{17}$ Dopaminergic cells have a more tonic output ${ }^{18}$ which could be used to influence the strength of such reference signals.

\section{Summary}

Evidence is presented that in bradykinesia and dyskinesia there is a mismatch of motor and sensory information. This is similar to the theory that appre- ciation of effort and weakness is through corollary discharges. It differs in that the mismatch is not consciously perceived and hence is not corrected. It has not been determined whether the corollary discharge or the sensory information is at fault.

I thank Professor J A Simpson, Dr I Bone and the referees for their helpful criticism, Dr S Hansen and Mr D Sutton for help with the equipment, Professor $M$ Titterington for statistical assistance, and to Sandoz Ltd for financial support.

\section{References}

1 Marsden CD. The mysterious motor function of the basal ganglia: the Robert Wartenberg lecture. Neurology 1982;32:514-39.

2 Hassler R. Striatal control of locomotion, intentional actions and of integrating and perceptive activity. $J$ Neurol Sci 1978;36:187-224.

3 Bodis-Wolner I, Yahr MD, Mylin LH. Non motor functions of the basal ganglia. In: Hassler RG, Christ JF, eds. Advances in Neurology 40: Parkinson Specific Motor and Mental Disorders. New York: Raven Press, 1984:289-98.

4 McCloskey I, Gandevia S, Potter EK, Colebatch JG. Muscle sense and effort: motor commands and judgments about muscular contractions. In: Desmedt J, ed. Advances in Neurology 39: Motor Control Mechanisms in Health and Disease. New York: Raven Press, 1983:151-67.

5 Matthews PBC. Where does Sherrington's muscular sense originate? Muscles, joints, corollary discharges? Ann Rev Neurosci 1982;5:189-218.

6 Goodwin GM, McCloskey DI, Matthews PBC. The contribution of muscle afferents to kinaesthesia shown by vibration induced illusions of movement and by the effects of paralyzing joint efferents. Brain 1972; 95:705-48.

7 Hughlings-Jackson J. Taylor J, ed. Selected Writings of John Hughlings-Jackson. Vol. 2. London: Hodder and Stoughton Ltd, 1932:422-43.

8 Cools AR, Jaspers R, Schwarz M, Sontag $K H$, Vrijmoed-de Vrien M, Van den Bercken J. Basal ganglia and switching motor programs. In: McKenzie JS, Kemm RE, Wilcock LN, eds. The Basal Ganglia: Structure and Function. New York: Plenum Press, 1984:513-35.

9 Bloxham CA, Mindel TA, Frith CD. Initiation and execution of predictable and unpredictable movements in Parkinson's disease. Brain 1984;107:371-84.

10 Angel RW. Efference copy in the control of movement. Neurology 1976;26:1164-8.

11 Draper IT, Johns RJ. The disordered movement in Parkinsonism and the effect of drug treatment. Bull Johns Hopkins Hosp 1964;115:465-80.

12 Tatton WG, Eastman MJ, Bedingham W, Verrier MC, Bruce IC. Defective utilization of sensory input as the basis for bradykinesia, rigidity and decreased movement repertoire in Parkinson's disease: a hypothesis. Can J Neurol Sci 1983;11:136-43. 
13 Dinnerstein AJ, Lowenthal M, Blake G, Mallin RE. Tactile delay in parkinsonism. $J$ Nerve Ment Dis 1964;139:521-4.

14 Gandevia SC, Rothwell JC. Subthreshold motor commands and the control of human motorneurones. Proc Physiological Society 1986:C36, 48.

15 DeLong MR, Alexander GE, Georgopoulos AP Crutcher MD, Mitchell SJ, Richardson RT. Role of basal ganglia in limb movements. Human Neurobiol 1984;2:235-44.

16 Bernardi G, Calabresi P, Mercuri N, Stanzione P. Dopamine decreases the amplitude of excitatory post- synaptic potentials in rat striatal neurones. In: McKenzie JS, Kemm RE, Wilcock LN, eds. The Basal Ganglia: Structure and Function. New York: Plenum Press, 1984:161-72.

17 McGeer EG, Staines WA, McGeer PL. Neuro- is transmitters in the basal ganglia. Can J Neurol Sci 1984;11:89-99.

18 Rolls ET. Responses of neurones in different regions of the striatum of the behaving monkey. In: McKenzie JS, Kemm RE, Wilcock LN, eds. The Basal Ganglia: Structure and Function. New York: Plenum Press, 1984:343-53. 